\title{
Symmetries of pp-Waves with Distributional Profile
}

\author{
Peter C. AICHELBURG 周 \\ Institut für Theoretische Physik, Universität Wien \\ Boltzmanngasse 5, A - 1090 Wien, AUSTRIA \\ and \\ Herbert BALASIN 宜由 \\ Institut für Theoretische Physik, Technische Universität Wien \\ Wiedner Hauptstraße 8-10, A - 1040 Wien, AUSTRIA
}

\begin{abstract}
We generalize the classification of (non-vacuum) pp-waves [1] based on the Killing-algebra of the space-time by admitting distributionvalued profile functions. Our approach is based on the analysis of the (infinite-dimensional) group of "normal-form-preserving" diffeomorphisms.
\end{abstract}

PACS numbers: 9760L, 0250

UWThPh $-1995-30$

TUW $95-20$

October 1995

\footnotetext{
* e-mail: pcaich @ pap.univie.ac.at

$\S$ supported in part by the FUNDACION FEDERICO

$\dagger$ e-mail: hbalasin@ email.tuwien.ac.at

${ }^{\ddagger}$ supported by the APART-program of the Austrian Academy of Sciences
} 


\section{1) Introduction}

In 1960, in a now classical paper, Jordan, Ehlers and Kundt (JEK) [1] gave a complete classification for the special class of gravitational waves with parallel rays ( pp-waves) in terms of their symmetries. These vacuum spacetimes which admit a non-shearing, non-twisting, and non-expanding null congruence can be characterized by the existence of a covariantly constant (cc) null vector field. In a recent paper the present authors [2] remarked that the JEK classification fails for the "gravitational field of a mass-less particle". This so-called AS-metric can be obtained by considering the ultra-relativistic limit of the Schwarzschild geometry [3, 4]. In the limit one is left with a ppwave whose profile-function is concentrated on a null hyper-plane. (Whereas in general a delta-like behavior of some of the metric components leads to illdefined quantities such as the Riemann-tensor, this is not so for the case under consideration.) A comparison of the AS-metric with the JEK-classification shows that this pp-wave should have only two Killing symmetries. By direct calculation however, it is easy to show that there are in fact four Killing vectors, as one would expect on physical grounds. For a detailed discussion see [2]. This discrepancy can be explained by the fact that in the JEKclassification the wave profile was tacitly assumed to be a classical (regular) function rather than a distribution.

Motivated by this we reconsider the JEK-classification by allowing for distributional wave profiles. Moreover, we allow for non-vacuum space-times (see also [5]), i.e. we do not impose any field equations from the outset. As a result we show that this generalization does indeed lead to additional symmetry groups for pp-waves, even in the vacuum case of which the ASmetric is one example.

\section{2) Normal-form-preserving diffeomorphisms and the characterization of pp-waves}

Since we do not want to impose Einstein's vacuum equations we define ppwaves by requiring the existence of a vector-field $p^{a}$ and a 2 -vector-field $F^{a b}$ such that $p^{[a} F^{b c]}=0$. A metric will be called pp-wave if $p^{a}$ is null and $p^{a}$ and $F^{a b}$ are mutually orthogonal and covariantly constant. In [1] it was shown, using adapted coordinates $\left(u, v, x^{i}\right)$, that any such pp-wave can be written 
in normal form

$$
d s^{2}=-d u d v+\delta_{i j} d x^{i} d x^{j}+f(u, x) d u^{2},
$$

where the cc vector is given by $\partial_{v}$ and the $x^{i}, i=1,2$ are coordinates on the flat $u=$ const, $v=$ const surfaces $\mathcal{S}$. However, for a given pp-wave the normal form does not fix the coordinate system uniquely. Transformations that do not change the form of the metric (1i) will be called normal-formpreserving (nfp). This subgroup of the full diffeomorphism-group consists of transformations of the form

$$
\begin{aligned}
& \tilde{u}=a u+b \quad \tilde{v}=\frac{1}{a}\left(v+2\left(\delta_{i j} \Omega^{i}{ }_{k} x^{k} d^{\prime j}(u)+n(u)\right)\right) \\
& \tilde{x^{i}}=\Omega^{i}{ }_{j} x^{j}+d^{i}(u)
\end{aligned}
$$

under which the profile function is changed into

$$
\begin{aligned}
\tilde{f}(u, x)=a^{2} f(a u+b, \Omega x+d(u))+\delta_{i j} d^{\prime}(u) d^{\prime j}(u) & \\
& -2\left(n^{\prime}(u)+\delta_{i j} \Omega^{i}{ }_{k} x^{k} d^{\prime \prime j}(u)\right),
\end{aligned}
$$

where $a$ and $b$ are constants, $\Omega$ is a constant rotation matrix, i.e. $\Omega^{t} \Omega=i d$, $d^{i}(u)$ denotes a vector tangential to $\mathcal{S}$ depending on $u$ and $n(u)$ is a scalar function of $u$. Prime denotes differentiation with respect to $u$. Thus, nfpdiffeomorphisms relating equivalent pp-waves form an infinite dimensional group with "parameters" $\left(a, b, \Omega^{i}{ }_{j} ; d^{i}(u), n(u)\right)$. The main difficulty in classifying pp-waves in terms of symmetries is finding a "canonical" representative for the profile functions. Since we would like to consider distributional wave profiles, it is natural to restrict to $C^{\infty}$-diffeomorphisms. (For a general definition of distributions on an arbitrary manifold, see [6].) Note that the nfp-transformations comprise possible isometries as a finite-dimensional subgroup, namely as those transformations that do not change the profile function. Let $\left(\alpha, \beta, \omega_{j}^{i} ; \theta^{i}(u), \nu(u)\right)$ be the corresponding parameters of an infinitesimal nfp-transformation. As a consequence the Killing-vectors $\xi$ may be written as

$$
\begin{aligned}
& \xi^{u}=\alpha u+\beta, \\
& \xi^{v}=-\alpha v+2\left(\theta^{\prime}(u) \cdot x+\nu(u)\right), \\
& \xi^{i}=\omega_{j}^{i} x^{j}+\theta^{i}(u),
\end{aligned}
$$


and the Killing condition restricts the profile to

$$
2 \alpha f+(\alpha u+\beta) f^{\prime}-x \omega \partial f+\theta(u) \partial f-2\left(\nu^{\prime}(u)+\theta^{\prime \prime}(u) \cdot x\right)=0 .
$$

From now on we will suppress the two-dimensional indices, using a matrixtype notation instead.

\section{3) Classification of Adjoint Orbits}

Since the nfp-group does not only act on profile functions $f$ but also on the space of (possible) Killing-vectors $\xi$, the latter action may rephrased in a purely group-theoretic setting without any reference to a specific profile. With regard to the notation of the previous chapter an arbitrary element $g$ of the nfp-group $G$ will be parametrised by

$$
g=(a, b, \Omega ; d(u), n(u)) .
$$

Composition of nfp-transformations allows us to define the multiplication map $\mu: G \times G \rightarrow G$ in parameter-space together with the adjoint action $k_{g}: G \rightarrow G$

$$
\begin{gathered}
g_{3}=\mu\left(g_{2}, g_{1}\right)=g_{2} \cdot g_{1} \\
a_{3}=a_{2} a_{1}, \quad b_{3}=a_{2} b_{1}+b_{2}, \quad \Omega_{3}=\Omega_{2} \Omega_{1}, \\
d_{3}(u)=\Omega_{2} d_{1}(u)+\phi_{1}^{*} d_{2}(u), \\
n_{3}(u)=n_{1}(u)+a_{1} \phi_{1}^{*} n_{2}(u)+a_{1}\left(\Omega_{2} d_{1}(u)\right) \cdot\left(\phi_{1}^{*} d_{2}^{\prime}(u)\right) \\
\text { where } \phi(u):=a u+b \quad\left(\phi^{*} f\right)(u):=f(a u+b) . \\
\tilde{h}=k_{g}(h):=g h g^{-1} \\
a_{\tilde{h}}=a_{h}, \quad b_{\tilde{h}}=a b_{h}-a_{h} b+b, \quad \Omega_{\tilde{h}}=\Omega_{h}, \\
d_{\tilde{h}}(u)=\phi^{-1 *}\left(-\Omega_{h} d(u)+\Omega d_{h}(u)+\phi_{h}^{*} d(u)\right), \\
n_{\tilde{h}}(u)=\frac{1}{a} \phi^{-1 *}\left[n_{h}(u)-\left(n(u)-d(u) \cdot d^{\prime}(u)\right)+a_{h} \phi_{h}^{*} n(u)+\right. \\
\left.a_{h}\left(\Omega d_{h}(u)\right) \cdot\left(\phi_{h}^{*} d^{\prime}(u)\right)-\left(\Omega_{h} d(u)\right) \cdot\left(\Omega d^{\prime}{ }_{h}(u)+a_{h} \phi_{h}^{*} d^{\prime}(u)\right)\right] .
\end{gathered}
$$


In order to find the adjoint representation of $G$ on its Lie-algebra, we consider an arbitrary curve through the identity $e=(1,0, i d ; 0,0)$ of the group and calculate its derivative at $e$. Parametrising a generic Lie-algebra-element by

$$
X=(\alpha, \beta, \omega ; \theta(u), \nu(u))
$$

the adjoint representation becomes

$$
\begin{aligned}
& \tilde{X}=A d(g) X, \\
& \tilde{\alpha}=\alpha, \quad \tilde{\beta}=a \beta-b \alpha, \quad \tilde{\omega}=\omega, \\
& \tilde{\theta}(u)=\phi^{-1 *}\left(\Omega \theta(u)-\omega d(u)+(\alpha u+\beta) d^{\prime}(u)\right), \\
& \tilde{\nu}(u)=\frac{1}{a} \phi^{-1 *}\left[\nu(u)+\alpha n(u)+(\alpha u+\beta) n^{\prime}(u)+(\Omega \theta(u)) \cdot d^{\prime}(u)-\right. \\
& \left.\quad d(u) \cdot\left(\Omega \theta^{\prime}(u)+\alpha d^{\prime}(u)+(\alpha u+\beta) d^{\prime \prime}(u)\right)-(\omega d(u)) \cdot d^{\prime}(u)\right] .
\end{aligned}
$$

The explicit form (7) of the adjoint representation allows us to classify its orbits. It turns out that there are eight different orbits. From each orbit we may obtain a particularly simple (canonical) representative by taking advantage of the adjoint transformations. We then require this representative to be the generator of an isometry. By solving the corresponding Killing equation the form of the profile function is obtained. The main advantage of this method lies in splitting the problem into a profile-dependent and independent part. We summarize our results in the following table.

a) $\omega \neq 0, \alpha \neq 0 \quad X=\left(1,0, \omega_{1} ; 0,0\right) \quad \omega_{1}=\frac{\omega}{\alpha}$

$$
u f^{\prime}+2 f=x \omega_{1} \partial f \Rightarrow f(u, x)=\frac{1}{u^{2}} \Phi\left(e^{-\omega_{1} \log u} x\right)
$$

b) $\omega \neq 0, \alpha=0, \quad X=\left(0,1, \omega_{1} ; 0,0\right) \quad \omega_{1}=\frac{\omega}{\beta}$

$$
\beta \neq 0 \quad f^{\prime}=x \omega_{1} \partial f \Rightarrow f(u, x)=\Phi\left(e^{-\omega_{1} u} x\right)
$$




$$
\begin{aligned}
& \text { c) } \omega \neq 0, \alpha=0, \quad X=\left(0,0, \omega_{1} ; 0, \nu(u)\right) \quad \omega_{1}=\omega \\
& \beta=0 \quad 2 \nu^{\prime}+x \omega_{1} \partial f=0 \Rightarrow f(u, x)=F\left(u, x^{2}\right) \quad \nu=\nu_{0} \\
& \text { d) } \omega=0, \alpha \neq 0, \quad X=\left(1,0,0 ; \theta_{1}, 0\right) \quad \theta_{1}=\frac{\theta(0)}{\alpha} \\
& \theta(0) \neq 0 \quad u f^{\prime}+2 f=-\theta_{1} \partial f \Rightarrow f(u, x)=\frac{1}{u^{2}} \Phi\left(x-\log u \theta_{1}\right) \\
& \text { e) } \omega=0, \alpha \neq 0, \quad X=(1,0,0 ; 0,0) \\
& \theta(0)=0 \quad u f^{\prime}+2 f=0 \Rightarrow f(u, x)=\frac{1}{u^{2}} \Phi(x) \\
& \text { f) } \omega=\alpha=0, \beta \neq 0 \quad X=(0,1,0 ; 0,0) \\
& f^{\prime}=0 \Rightarrow f(u, x)=\Phi(x) \\
& \text { g) } \omega=\alpha=0, \quad X=(0,0,0 ; \theta(u), \nu(u)) \\
& \beta=0, \theta(u) \neq 0 \quad-\theta(u) \partial f+2 \theta^{\prime \prime}(u) \cdot x+2 \nu^{\prime}(u)=0 \\
& \text { h) } \omega=\alpha=0 \quad X=(0,0,0 ; 0, \nu(u)) \\
& \beta=\theta(u)=0 \quad \nu^{\prime}=0 \Rightarrow f(u, x)=f(u, x)
\end{aligned}
$$

Different orbits are characterized by the invariant group parameters $\alpha$ and $\omega$ and split into sub-cases depending on the other parameters. The representative $X$ of each orbit, the Killing equation and the corresponding form of the profile function $f$ are given. The explicit form of the Killing fields is obtained by inserting $X$ into (3). Case g) may be classified further with respect to the number and the degree of the zeros of $\theta(u)$. The simplest case, $\theta(u)$ without any zeros, reproduces the result of [2] (after imposing vacuum equations), whereas the others allow concentrated (delta-like) contributions to the profile. Finally, case h) is the generic pp-wave with one Killing vector, without any restriction on the profile $f$. Thus each class $(\mathrm{a}-\mathrm{g})$ admits $\partial_{v}$ as Killing vector. Symmetry groups of three or more parameters can be obtained by combining the above cases. Note however, that this is not straightforward since it is not guaranteed that the Killing vectors can be reduced to their canonical form simultaneously. A complete analysis of this problem will be published in an more elaborated paper. Here we restrict ourselves to discuss pp-waves with delta-like profiles. 


\section{4) Impulsive pp-waves}

Let us focus our attention on profiles of the form

$$
f(u, x)=\delta(u) f(x),
$$

since they are natural candidates for a richer symmetry structure. In order to classify these space-times we follow exactly the same path as in the general case: We consider those nfp-transformations, which preserve (8). Following (4) the action of an arbitrary nfp-transformation on (8) yields

$$
\begin{aligned}
\tilde{f}(x, u)=a^{2} \delta(a u+b) f( & \Omega x+d(u)) \\
& +d^{\prime}(u)^{2}-2\left(n^{\prime}(u)+\Omega x \cdot d^{\prime \prime}(u)\right)=: \delta(u) \tilde{f}(x) .
\end{aligned}
$$

The $C^{\infty}$ nature 1 of the nfp-transformations and the location of the singularity require $d^{\prime \prime}(u)=0,2 n^{\prime}(u)-d^{\prime}(u)^{2}=0, b=0$. Therefore the nfp-group is cut down to a finite-dimensional subgroup $G_{0}$

$$
\begin{aligned}
& g=\left(a, 0, \Omega ; d_{0}+u d_{1}, 1 / 2 u d_{1}^{2}+n_{0}\right), \\
& X=\left(\alpha, 0, \omega ; \theta_{0}+u \theta_{1}, \nu_{0}\right), \\
& \tilde{f}(x)=a f\left(\Omega x+d_{0}\right),
\end{aligned}
$$

which will be called restricted normal-form-preserving (rnfp) group. An immediate consequence of $(10)$ is the fact that $\tilde{f}$ does not depend on $d_{1}$. This implies that all impulsive waves admit a three-parameter Killing-group in contrast to the one-parameter group for general profiles. The adjoint representation of the rnfp-group is easily derived from (7)

$$
\begin{aligned}
& X=\left(\alpha, 0, \omega ; \theta_{0}+u \theta_{1}, \nu_{0}\right) \quad \tilde{X}=A d(g) X \quad g \in G_{0} \\
& \tilde{\alpha}=\alpha, \quad \tilde{\omega}=\omega \\
& \tilde{\theta_{0}}=\Omega \theta_{0}-\omega d_{0} \quad \tilde{\theta_{1}}=\frac{1}{a}\left(\Omega \theta_{1}-\omega d_{1}+\alpha d_{1}\right) \\
& \tilde{\nu_{0}}=\frac{1}{a}\left(\nu_{0}+\alpha n_{0}+\Omega \theta_{0} \cdot d_{1}-d_{0} \cdot \Omega \theta_{1}-\omega d_{0} \cdot d_{1}-\alpha d_{0} \cdot d_{1}\right)
\end{aligned}
$$

Taking into account that $\theta_{1}$ and $\nu_{0}$ are already Killing parameters, i.e. that they do not change the profile, it is possible to simplify (11):

$$
\begin{aligned}
& X=\left(\alpha, 0, \omega ; \theta_{0}, 0\right) \quad g=\left(a, 0, \Omega ; d_{0}, 0\right) \\
& \tilde{\alpha}=\alpha, \quad \tilde{\omega}=\omega, \quad \tilde{\theta_{0}}=\Omega \theta_{0}-\omega d_{0}
\end{aligned}
$$

\footnotetext{
${ }^{1}$ Actually, the result remains unchanged if one admits "distributional" $\left(C^{0}\right)$ coordinatechanges
} 
And finally the Killing-equation is reduced to

$$
0=\alpha f(x)-x \omega \partial f(x)+\theta_{0} \partial f(x),
$$

which restricts the profile $f(x)$. The analogous classification to (a)-h)) is now obtained in a straightforward way:

1) $\omega \neq 0, \alpha=0$ : Using the adjoint action (11) it is possible to reduce the representative to $X=(0,0, \omega ; 0,0)$, turning the Killing-condition into $x \omega \partial f(x)=0 \Rightarrow f(x)=g\left(x^{2}\right)$.

2) $\omega=0, \alpha \neq 0$ : Since the Killing-vector is unique up to scalar multiple, we set $\alpha=1$, which leaves us with $X=\left(1,0,0 ; \theta_{0}, 0\right)$ and $\left(\theta_{0} \partial\right) f=$ $-f \Rightarrow f(x)=h\left(\tilde{\theta}_{0} x\right) e^{-\frac{\theta_{0} x}{\theta_{0}^{2}}}$, where $\tilde{\theta}_{0}$ denotes the dual with respect to the induced metric on $\mathcal{S}$.

3) $\omega=\alpha=0$ : The representative in this orbit becomes $X=\left(0,0,0 ; \theta_{0}, 0\right)$, which implies $\theta_{0} \partial f=0 \Rightarrow f(x)=h\left(\tilde{\theta}_{0} x\right)$, where the same notation is used as in the cases above.

4) $\omega \neq 0, \alpha \neq 0$ : This case requires some discussion. Using polarcoordinates $(\rho, \phi)$ and the form of the representative $X=(1,0, \omega ; 0,0)$, we find $f=\gamma \partial_{\phi} f \quad\left(\gamma=\frac{1}{2} \epsilon^{i j} \omega_{i j}\right) \Rightarrow f(x)=h(\rho) e^{\gamma \phi}$, which is not globally defined on $\mathcal{S}$. Unwrapping $\mathcal{S}$ with the help of

$$
w=\log z \quad p+i q=\log \rho+i \phi
$$

$f(x)$ becomes a well-defined function on the infinite cover of $\mathcal{S}$, i.e. $f(p, q)=h\left(e^{p}\right) e^{\gamma q}$.

All of the above cases possess a four-parametric Killing-algebra. Imposing the vacuum-equations turns 1) into the AS-geometry, the arbitrary function $h$ in 2) and 4) become harmonic and 3) reduces to a linear profile thus being equivalent to Minkowski-space. Higher symmetry-classes may be obtained by combining the above classes. However, like in the general case, one cannot assume that both Killing-vectors (and the corresponding profiles) are simultaneously in their canonical form. In general one has to apply an arbitrary rnfp-transformation to the canonical form of the profile relative to the first Killing-vector, and impose the second Killing afterwards. Taking the respective stability-(sub)groups of both Killing-vectors into account it is 
possible to simplify the arbitrary rnfp-transformation considerably. Applying this procedure to 1)-4) it turns out that only the combination of 2) and 3) yields a non-trivial result.

$$
\begin{aligned}
& f(x)=h_{0} \exp \left(\frac{1}{\theta_{0}^{2}}\left(\frac{\theta_{0} \eta_{0}}{\tilde{\theta}_{0} \eta_{0}} \tilde{\theta_{0}} x-\theta_{0} x\right)\right) \\
& X=\left(1,0,0 ; \theta_{0}, 0\right) \quad Y=\left(0,0,0 ; \eta_{0}, 0\right)
\end{aligned}
$$

Note however that the symmetries of (13) are not compatible with Einstein's vacuum equations. Let us finally summarize the results in the following table:

\begin{tabular}{|l|l|l|l|}
\hline profile $f(x, y)$ & Killing-vectors $\xi$ & $\mathrm{r}$ & type \\
\hline \hline general & $\xi_{1}=\partial_{v}, \xi_{2}=2 x \partial_{v}+u \partial_{x}$, & 3 & abelian \\
& $\xi_{3}=2 y \partial_{v}+u \partial_{y}$ & & \\
\hline$g\left(x^{2}+y^{2}\right)$ & $\xi_{1}, \xi_{2}, \xi_{3}, \xi_{4}=x \partial_{y}-y \partial_{x}$ & 4 & $E_{2} \times \mathbb{R}$ \\
\hline$h(y) e^{-x / \epsilon}$ & $\xi_{1}, \xi_{2}, \xi_{3}, \xi_{4}=u \partial_{u}-v \partial_{v}+\epsilon \partial_{x}$ & 4 & {$\left[\xi_{4}, \xi_{1}\right]=\xi_{1}$,} \\
& & & {$\left[\xi_{4}, \xi_{2}\right]=\xi_{2}+2 \epsilon \xi_{1}$,} \\
& & & {$\left[\xi_{4}, \xi_{3}\right]=\xi_{3}$} \\
\hline$h(y)$ & $\xi_{1}, \xi_{2}, \xi_{3}, \xi_{4}=\partial_{x}$ & {$\left[\xi_{4}, \xi_{1}\right]=0$,} \\
& & & {$\left[\xi_{4}, \xi_{2}\right]=2 \xi_{1}$,} \\
& & $\left.4 \xi_{4}, \xi_{3}\right]=0$ \\
\hline$h\left(e^{p}\right) e^{\gamma q}$ & $\xi_{1}, \xi_{2}, \xi_{3}$, & {$\left[\xi_{4}, \xi_{1}\right]=\xi_{1}$,} \\
& $\xi_{4}=u \partial_{u}-v \partial_{v}+\gamma\left(x \partial_{y}-y \partial_{x}\right)$ & & {$\left[\xi_{4}, \xi_{2}\right]=\xi_{2}-\gamma \xi_{3}$,} \\
& & & {$\left[\xi_{4}, \xi_{3}\right]=\xi_{3}+\gamma \xi_{2}$} \\
\hline$h_{0} e^{\frac{1}{\epsilon_{1}}\left(\frac{y}{\epsilon_{2}}-x\right)}$ & $\xi_{1}, \xi_{2}, \xi_{3}, \xi_{4}=u \partial_{u}-v \partial_{v}+\epsilon_{1} \partial_{x}$ & 5 & {$\left[\xi_{4}, \xi_{1}\right]=\xi_{1}$,} \\
& $\xi_{5}=\partial_{x}+\epsilon_{2} \partial_{y}$ & {$\left[\xi_{4}, \xi_{2}\right]=\xi_{2}+\epsilon_{1} \xi_{1}$,} \\
& & & {$\left[\xi_{4}, \xi_{3}\right]=\xi_{3}$,} \\
& & & {$\left[\xi_{5}, \xi_{1}\right]=0$,} \\
& & & {$\left[\xi_{5}, \xi_{2}\right]=2 \xi_{1}$,} \\
& & & {$\left[\xi_{5}, \xi_{3}\right]=2 \epsilon_{2} \xi_{1}$,} \\
& & \\
& & & \\
& &
\end{tabular}




\section{Conclusion}

In this paper we have generalized the classification of pp-waves in terms of Killing symmetries by allowing the profile function to be a delta-like pulse. Moreover, no field equations were imposed. The method presented relies on the analysis of the adjoint orbits of the so called normal-form-preserving diffeomorphisms. Our calculations show that new symmetry classes occur even in the vacuum case. In a forthcoming paper we intend to give a more detailed presentation for general wave profiles. There the analysis is considerably more involved. Nevertheless, we feel that the method presented may also be useful for classifying other types of space-times. 


\section{References}

[1] Jordan P Ehlers J and Kundt W, Akad. Wiss. Lit. (Mainz) Abhandl. Math.-Nat. Kl. 2, 21 (1960).

[2] Aichelburg P and Balasin H,Class. Quantum Grav. 11, L121 (1994).

[3] Aichelburg P and Sexl R, Gen. Rel. Grav. 2 (1971) 303.

[4] Balasin H and Nachbagauer H, Class. Quantum Grav. 12, 707 (1995).

[5] Sippel R and Goenner H, Gen. Rel. Grav. 18, 1229 (1986).

[6] Balasin H and Nachbagauer H, Class. Quantum Grav. 10, 2271 (1993). 